\title{
THE REACTION OF LOW-MASS STARS TO ANISOTROPIC IRRADIATION AND ITS IMPLICATIONS FOR THE SECULAR EVOLUTION OF CATACLYSMIC BINARIES
}

\author{
H. RITTER, Z. ZHANG AND U. KOLB \\ Max-Planck-Institut für Astrophysik \\ Karl-Schwarzschild-Str. 1 \\ Postfach 1523 \\ 85740 Garching, Germany
}

\begin{abstract}
A semi-analytic model for the reaction of a low-mass star to anisotropic irradiation of low incident flux is presented. By applying this model to the donor star of cataclysmic binaries (CBs) it is shown that CBs are likely to be unstable against irradiation-driven runaway mass transfer. The implications of this instability for the long-term evolution of CBs are examined. The possibility is discussed that because of this instability CBs evolve through a limit cycle in which phases of high and low mass transfer rate alternate on a time scale short compared to the evolutionary time scale.
\end{abstract}

\section{Introduction}

The possible importance of the reaction of low-mass stars to external irradiation for the long-term evolution of compact binaries has been realized only rather recently in the context of the evolution of low-mass X-ray binaries (LMXBs). So far, most papers have dealt with this problem in spherical symmetry and in the limit of the very high incident fluxes relevant for LMXBs (Podsiadlowski 1991; Harpaz \& Rappaport 1991; Frank et al. 1992; Hameury et al. 1993). The limit of low flux, but still in spherical symmetry, has been considered by D'Antona \& Ergma (1993). Anisotropic irradiation in the limit of high flux has been studied by Gontikakis \& Hameury (1993), Hameury et al. (1993) and by Ritter (1994). To the best of our knowledge, 
anisotropic irradiation in the limit of low flux has not been considered so far. In this paper we present a first attempt to do so. For this we develop a simple semi-analytical model for the reaction of a low-mass star to anisotropic irradiation and apply this model mainly to CBs.

\section{The Reaction of a Low-Mass Star to Anisotropic Irradiation}

The following discussion applies only to low-mass stars $\left(M \lesssim 1 \mathrm{M}_{\odot}\right)$ on or near to the main sequence. Such stars have either a deep outer convective envelope and a radiative core $\left(M>M_{\text {conv }} \approx 0.35 \mathrm{M}_{\odot}\right)$ or they are fully convective $\left(M<M_{\text {conv }}\right)$. We model the influence of anisotropic irradiation on the structure of such stars as follows: We assume that a fraction $s$ of the stellar surface is exposed to an incident flux $F_{\text {irr }}$ and that the remaining fraction $(1-s)$ of the surface remains in the shadow. Denoting by $R_{\mathrm{s}}$ the radius of the star and by $T_{1}$ and $T_{2}$ respectively the effective temperatures on the unlit and the irradiated part, the stellar luminosity (energy lost by the star from its interior per unit time) is

$$
L=4 \pi R_{\mathrm{s}}^{2}\left\{(1-s) \sigma T_{1}^{4}+s \sigma T_{2}^{4}-s F_{\mathrm{irr}}\right\} .
$$

In order to use the modified Stefan-Boltzmann law (1) as an outer boundary condition for stellar evolution calculations one has to know $T_{2}$. This, in turn, is determined by the details of how irradiation affects the energy loss through the irradiated surface.

Now it is important to realize that because the stars under investigation have a deep outer adiabatic convective envelope their mechanical and thermal structure remains spherically symmetric to a very high degree of accuracy out to the onset of the superadiabatic convection zone despite anisotropic irradiation. It is only the very thin superadiabatic layer (mass $\sim 10^{-10} \mathrm{M}_{\odot}$ ), where energy is mainly transported via radiation, which is strongly affected by irradiation. So the asphericity of the star is restricted to a very thin surface layer, and it is this property which allows us to determine $T_{2}$ in (1) with a simple model. In this model we assume that convection is adiabatic out to a point where the pressure is $P=P_{\mathrm{B}}$ and the temperature $T=T_{\mathrm{B}}$ (see the sketch in Fig. 1).

The point $\left(P_{\mathrm{B}}, T_{\mathrm{B}}\right)$ is the base of the superadiabatic convection zone which extends to the photosphere where the pressure is the photospheric pressure $P_{\mathrm{ph}}$ and the temperature the effective temperature $T_{\text {eff. }}$. Because in this zone convection is ineffective, we assume the energy transport to be only radiative, i.e. $\nabla_{\mathrm{rad}}=\nabla$ (where $\nabla \equiv \partial \ln T / \partial \ln P$ ). Because on the irradiated part of the star the effective temperature is $T_{2}>T_{1}$, but $T_{\mathrm{B}}$ is the same for both parts, we see that irradiation, by raising the effective temperature, reduces the temperature gradient $\nabla$ and thus the radiative 


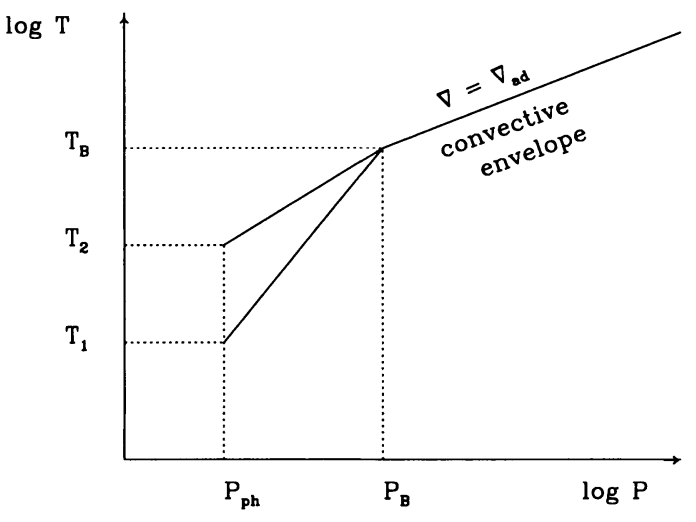

Figure 1. Sketch of the assumed run of temperature versus pressure in the outer layer of our model star.

energy loss through these layers. As is sketched in Fig. 1 we assume for simplicity that $P_{\mathrm{ph}}$ is the same on both parts of the star, though this is of course not strictly true. Using now the radiative diffusion approximation

$$
F_{\mathrm{rad}}=-\frac{a c}{3 \kappa \varrho} \frac{d T^{4}}{d r}
$$

where $\varrho$ is the density, $\kappa$ the opacity, and the other symbols have their usual meaning, and a power law approximation for $\kappa$

$$
\kappa=\text { const. } P^{a} T^{b}
$$

a one zone model for the superadiabatic layers yields (for details see Ritter et al. 1995)

$$
\frac{F_{\mathrm{rad}, 1}}{F_{\mathrm{rad}, 2}} \equiv \frac{\sigma T_{1}^{4}}{\sigma T_{2}^{4}-F_{\mathrm{irr}}}=\left\{\begin{array}{ll}
\frac{T_{\mathrm{B}}^{n}-T_{1}^{n}}{T_{\mathrm{B}}^{n}-T_{2}^{n}}, & n=5-b \neq 0 \\
\frac{\ln T_{\mathrm{B}}-\ln T_{1}}{\ln T_{\mathrm{B}}-\ln T_{2}} & , \quad n=5-b=0
\end{array} .\right.
$$

Eq. (4) can be solved for $T_{2}\left(T_{1}, T_{\mathrm{B}}, F_{\text {irr }}\right)$ which together with Eq. (1) can be used as an outer boundary condition for numerical calculations.

\section{Stability against Irradiation-Induced Mass Transfer}

Let's now examine the situation in which a low-mass star transfers mass to a compact companion and, in turn, is irradiated by the accretion light source. For reasons which will become clear later, we restrict our discussion 
mainly to CBs, i.e. the compact star is a white dwarf (of mass $M_{\mathrm{WD}}$ and radius $R_{\mathrm{WD}}$ ). The average flux with which the donor star is irradiated is

$$
\left\langle F_{\mathrm{irr}}\right\rangle=\frac{\eta}{8 \pi} \frac{G M_{\mathrm{WD}}\left(-\dot{M}_{\mathrm{s}}\right)}{R_{\mathrm{WD}} a^{2}},
$$

where $\left(-\dot{M}_{\mathrm{s}}\right)$ is the mass transfer rate, $a$ the orbital separation and $\eta \lesssim 1$ a dimensionless "efficiency" factor which absorbs such factors as the albedo of the donor and takes into account that the irradiating light source does not necessarily radiate isotropically. Now, from Eqs. (1) and (4) one derives that

$$
\frac{\partial L}{\partial F_{\mathrm{irr}}}=-4 \pi R_{\mathrm{s}}^{2} s g\left(T_{1}, T_{2}, T_{\mathrm{B}}\right)<0
$$

where

$$
g\left(T_{1}, T_{2}, T_{\mathrm{B}}\right)= \begin{cases}\frac{n T_{1}^{4} T_{2}^{n-1}}{n T_{1}^{4} T_{2}^{n-1}+4 T_{2}^{3}\left(T_{\mathrm{B}}^{n}-T_{1}^{n}\right)}, & n=5-b \neq 0, T_{\mathrm{B}}>T_{2} \\ \frac{T_{1}^{4}}{T_{1}^{4}+4 T_{2}^{4}\left(\ln T_{\mathrm{B}}-\ln T_{1}\right)}, & n=5-b=0, T_{\mathrm{B}}>T_{2} .\end{cases}
$$

Thus the main effect of irradiating the star is to reduce its luminosity. This, in turn, means that part of the energy which the star generates in its interior is prevented from leaking through the irradiated surface and, therefore, is stored as internal and gravitational energy with the result that the star swells. In a mass-transferring binary, the swelling of the donor drives additional mass transfer and in this way generates even more irradiating flux. Therefore, we need now to examine under which conditions such a situation is stable against irradiation-induced runaway mass transfer. For this we write the temporal change of the stellar radius as

$$
\frac{d \ln R_{\mathrm{s}}}{d t}=\zeta_{S} \frac{\dot{M}_{\mathrm{s}}}{M_{\mathrm{s}}}+\left(\frac{\partial \ln R_{\mathrm{s}}}{\partial t}\right)_{\mathrm{ml}}+\left(\frac{\partial \ln R_{\mathrm{s}}}{\partial t}\right)_{\mathrm{irr}},
$$

where $\zeta_{S}$ is the adiabatic mass radius exponent, $\left(\partial \ln R_{\mathrm{s}} / \partial t\right)_{\mathrm{ml}}$ the thermal relaxation term due to mass loss and $\left(\partial \ln R_{\mathrm{s}} / \partial t\right)_{\text {irr }}$ the one due to irradiation. The condition for (dynamical) stability against mass transfer can be derived in the same way as, e.g., in Ritter (1988). The result is

$$
\begin{aligned}
\zeta_{S}-\zeta_{R}>\zeta_{\text {irr }} & \equiv-M_{\mathrm{s}} \frac{\partial}{\partial \dot{M}_{\mathrm{s}}}\left(\frac{\partial \ln R_{\mathrm{s}}}{\partial t}\right)_{\mathrm{irr}} \\
& =-M_{\mathrm{s}} \frac{\partial}{\partial L}\left(\frac{\partial \ln R_{\mathrm{s}}}{\partial t}\right)_{\mathrm{irr}} \frac{\partial L}{\partial F_{\mathrm{irr}}} \frac{\partial F_{\mathrm{irr}}}{\partial M_{\mathrm{s}}},
\end{aligned}
$$

where $\zeta_{R}$ is the mass radius exponent of the Roche radius, and $\zeta_{\text {irr }}$ is a dimensionless number which measures how sensitively the stellar radius 


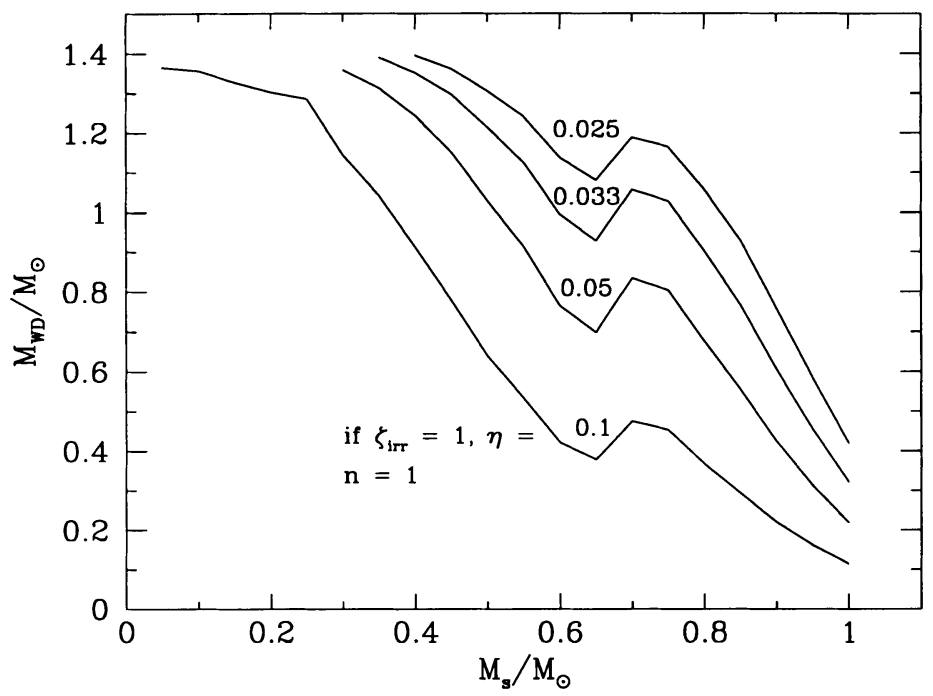

Figure 2. Contour lines for $\eta=$ const. in the $M_{\mathrm{s}}-M_{\mathrm{WD}}$ plane along which $\zeta_{\text {irr }}=1$. The donor star is assumed to be on the main sequence with $T_{2}=T_{1}, s=0.5$ and $n=1$ $\left(\hat{=} b=4\right.$, i.e. $\mathrm{H}^{-}$opacity).

changes in response to irradiation. In order to evaluate $\partial^{2} \ln R_{\mathrm{s}} / \partial L \partial t$ we use the bipolytrope model (e.g., Kolb \& Ritter 1992) and obtain

$$
\begin{aligned}
& \frac{\partial}{\partial L}\left(\frac{\partial \ln R_{\mathrm{s}}}{\partial t}\right)_{\mathrm{irr}}= \\
& -\frac{R_{\mathrm{s}}}{G M_{3}^{2}}\left\{\frac{\partial H}{\partial Q} \frac{1}{H_{2}\left(Q, n_{1}\right)}+\frac{1}{f} \frac{\partial f}{\partial Q}\right\}\left\{\frac{\partial H}{\partial Q}+\frac{H}{f} \frac{\partial f}{\partial Q}\right\}^{-1},
\end{aligned}
$$

where the quantities in the curly brackets \{\}\{\}$^{-1}$ define a dimensionless number which depends only on the relative size $Q=r_{\text {core }} / R_{\mathrm{s}}$ of the radiative core and the polytropic index $n_{1}$ in it. In particular, for a single polytrope $n=3 / 2$, i.e. $Q=0$, one has \{\}\{\}$^{-1}=7 / 3$. Taking now $F_{\text {irr }}=\left\langle F_{\text {irr }}\right\rangle$, Eqs $(5,6,7)$ yield

$$
\zeta_{\mathrm{irr}}=\frac{\eta}{2} \frac{M_{\mathrm{WD}}}{M_{\mathrm{s}}} \frac{R_{\mathrm{s}}}{R_{\mathrm{WD}}}\left(\frac{R_{\mathrm{s}}}{a}\right)^{2} s\{\}\{\}^{-1} g\left(T_{1}, T_{2}, T_{\mathrm{B}}\right) .
$$

Since in a normal CB $\zeta_{S}-\zeta_{R} \approx 1$, systems in which $\zeta_{\text {irr }} \gtrsim 1$ are unstable against irradiation-induced runaway mass transfer. Under which conditions is $\zeta_{\text {irr }}>1$ ? Using ZAMS models for the donor (to determine $R_{\mathrm{s}}\left(M_{\mathrm{s}}\right)$ and $T_{\mathrm{B}}\left(M_{\mathrm{s}}\right)$ ), Nauenberg's (1972) mass-radius relation for white dwarfs and $T_{2}=T_{1}$ (initial state is an unirradiated star) we find that $\zeta_{\text {irr }}>1$ is reached 
for surprisingly small values of $\eta$ (see Fig. 2), i.e. $\eta \gtrsim 0.04 \ldots 0.1$ is sufficient for instability. The conclusion to be drawn from this is that at least a large fraction of CBs is likely to be unstable against irradiation-induced runaway mass transfer.

Before we proceed to discuss the long-term evolution of CBs under this instability, we can now make a brief remark regarding the LMXBs. Replacing the white dwarf by a neutron star means that, if everything else remains equal, $\zeta_{\text {irr }}$ is larger by a factor $R_{\mathrm{WD}} / R_{\mathrm{NS}} \gtrsim 10^{3}$ and that, therefore, $\zeta_{\text {irr }} \gtrsim 1$ is reached for much smaller $\eta$, i.e. $\eta \gtrsim 10^{-3}$. Thus LMXBs are extremely unstable against irradiation of the donor star.

\section{Long-Term Evolution of CBs under the Irradiation Instability}

Since there is no observational evidence showing that the known CBs are in a phase of runaway mass transfer and since a number of well-observed properties of CBs such as the period gap can only be explained if their evolution on the long-term average follows closely a standard evolution without taking into account irradiation (e.g. Kolb \& Ritter 1992; Kolb 1993), we must conclude that the instability is either not relevant for CBs (because $\eta$ is too small) or, if it is, that the instability must be quenched such as to prevent the mass transfer from running away too strongly. If the latter is the case, it is then conceivable that CBs go through a limit cycle in which phases of high and low states alternate on a time scale short compared to the evolutionary time scale. The high state would correspond to a phase during which the donor swells in response to irradiation and transfers mass at a rate above the secular mean. Mass transfer above the secular mean, however, cannot be maintained indefinitely and sooner or later the system has to go into a low state during which the mass transfer rate is below the long-term mean and irradiation is correspondingly unimportant. The question, therefore, is whether there are such quenching mechanisms? In fact there are, and in the following we are going to describe two of them.

One quenching mechanism operates only for donor stars with a mass $M_{\mathrm{s}} \gtrsim \mathbf{0 . 6 \mathrm { M } _ { \odot }}$. In this case quenching is possible because with increasing $T_{2}$ not only the temperature gradient decreases but also the optical depth in the superadiabatic layer becomes larger (note that the relevant opacity source is $\mathrm{H}^{-}$and the corresponding value of $b$ is $\left.b \approx 4-5\right)$. As a consequence of this $\partial \zeta_{\text {irr }} / \partial T_{2}<0$. Numerical calculations of the long-term evolution of systems with a donor in the mass range $0.6 \mathrm{M}_{\odot} \lesssim M_{s} \lesssim 0.9 \mathrm{M}_{\odot}$ show in fact that the systems can go through a limit cycle, though the oscillations are damped on a time scale of $\sim 10^{7} \mathrm{yr}$. Details will be presented elsewhere (Ritter et al. 1995).

The other quenching mechanism is more fundamental. Quenching sets 
in as soon as $T_{2}=T_{\mathrm{B}}$. Since for stars with a mass $M \lesssim 0.6 \mathrm{M}_{\odot}$ already $T_{1}$ is only slightly lower than $T_{\mathrm{B}}, T_{2}=T_{\mathrm{B}}$ is reached for rather small mass transfer rates. As soon as $T_{2} \geq T_{\mathrm{B}}$, the star's luminosity $L$ does no longer depend on $F_{\text {irr }}$, i.e. $\partial L / \partial F_{\text {irr }}=0$ and so $\zeta_{\text {irr }}=0$. This is because for $T_{2} \geq T_{\mathrm{B}}$ an isothermal layer is formed on the irradiated side of the star which extends from the photosphere $\left(P=P_{\mathrm{ph}}\right)$ down to $P \approx P_{\mathrm{B}}\left(T_{2} / T_{\mathrm{B}}\right)^{1 / \nabla_{\mathrm{a}}}$, and through which no energy is transported, i.e. $\sigma T_{2}^{4}=F_{\text {irr. }}$. The mass transfer rate for which quenching sets in, i.e. when $T_{2}=T_{\mathrm{B}}$ is

$$
\begin{aligned}
\dot{M}_{\mathrm{q}} & =\frac{1}{\zeta_{\text {irr }}} \frac{s}{1-s} \frac{R_{\mathrm{s}} L}{G M_{\mathrm{s}}^{2}}\{\}\{\}^{-1}\left(\frac{T_{\mathrm{B}}}{T_{1}}\right)^{4} g\left(T_{1}, T_{2}=T_{\mathrm{B}}, T_{\mathrm{B}}\right) \\
& \lesssim \frac{s}{1-s} \frac{R_{\mathrm{s}} L}{G M_{\mathrm{s}}^{2}}\{\}\{\}^{-1} .
\end{aligned}
$$

When compared with the mass transfer rates for a standard CB evolution (e.g., Kolb \& Ritter 1992) we find that $\dot{M}_{\mathrm{q}}$ is less than $\dot{M}_{\mathrm{tr}}$, but by not more than a factor of two, if $M_{\mathrm{s}} \lesssim 0.5 \mathrm{M}_{\odot}$. This, in turn, would mean that no limit cycle is possible below $\sim 0.5 \mathrm{M}_{\odot}$. Rather such systems are stable and the irradiation instability permanently quenched, i.e. $T_{2}>T_{\mathrm{B}}$ at all times. However, as far as stars below $0.5 \mathrm{M}_{\odot}$ are concerned, our conclusions may be premature. This is because application of our model to stars with a mass $M \lesssim 0.6 \mathrm{M}_{\odot}$ is problematic. The reason is that Eq. (4) was derived in the diffusion approximation which requires that the optical depth in the superadiabatic layer is sufficiently high. This, however, is not the case if $T_{\mathrm{B}}$ is only slightly above $T_{1}$ as in main sequence stars with $M \lessgtr 0.6 \mathrm{M}_{\odot}$.

Thus it remains to be demonstrated whether the irradiation instability described here, in combination with a suitable quenching mechanism, allows for a stable and large amplitude limit cycle which, in turn, could account for the observed large scatter of the mass transfer rates of CBs around the long-term mean.

\section{References}

D'Antona, F. \& Ergma, E. 1993, A\&A 269, 219

Frank, J., King, A.R. \& Lasota, J.P. 1992, ApJ 385, L45

Gontikakis, C. \& Hameury, J.-M. 1993, A\&A 271, 118

Harpaz, A. \& Rappaport, S. 1991, ApJ 383, 739

Hameury, J.-M., King, A.R., Lasota, J.P. \& Raison, F. 1993 A\&A 277, 81

Kolb, U. 1993, A\&A 271, 149

Kolb, U. \& Ritter, H. 1992, A\&A 254, 213

Nauenberg, M. 1972, ApJ 175, 417

Podsiadlowski, Ph. 1991, Nat 350, 136

Ritter, H. 1988, A\&A 202, 93

Ritter, H. 1994, in Evolutionary Links in the Zoo of Interacting Binaries, F. D'Antona (Ed.), Mem. Soc. Astron. It., (in press)

Ritter, H., Zhang, Z. \& Kolb, U. 1995, (in preparation) 